\title{
Study on mode mixing problem of empirical mode decomposition
}

\author{
Guimin Xua* , Zhengxiang Yang ${ }^{b}$, Sha Wanga \\ aEngineering and Technology College, Hubei University of Technology, Wuhan 430068, China; \\ ${ }^{b}$ Department of Electronics and Information, Wuhan Technical College of Communications, \\ Wuhan 430065, China \\ ${ }^{*}$ Corresponding author. E-mail: bingfeng71@126.com
}

Keywords: empirical mode decomposition; mode mixing; intrinsic mode function

\begin{abstract}
After defined the basic concept of mode or intrinsic mode, EMD as a data-driven adaptive decomposition method, its purpose is to decompose arbitrary non-stationary and nonlinear signal into different intrinsic mode functions (IMFs) on the time series. However, in some cases, the EMD method often cannot get ideal decomposition results which can result mode mixing problem. once mode mixing phenomenon appear, mode mixing will affect the subsequent decomposition components, the time-frequency distribution of subsequent IMFs are confusion, eventually the EMD decomposition process lose physical meaning. The EMD mode mixing problem directly affect its application in various fields. This paper discuss the basic cause of mode mixing, the results show that: the jumping change of the decomposed signal result in mode mixing, mode mixing caused by amplitude and frequency relationship of the signal.
\end{abstract}

\section{Introduction}

Conventional data processing techniques, such as short time Fourier transform (STFT), wavelet transform (WT) and time-domain statistical analysis, have been used to analyze non-linear and non-stationary data ${ }^{[1-3]}$. These techniques are based on the assumption that the processed data are linear and stationary, which usually results in false information when dealing with non-linear and non-stationary data.

As an adaptive time frequency analysis method, Empirical mode decomposition (EMD) was proposed for processing non-linear and non-stationary signals ${ }^{[4]}$. Unlike wavelet based techniques, EMD has a distinct advantage that its functions are known as intrinsic mode functions (IMFs) adaptively from the signal itself. However, there are some annoying difficulties unresolved in EMD. The main shortcoming of EMD method is that it is prone to mode mixing, which is defined as a single IMF either consisting of widely disparate scales, or similar scale residing in different IMF components, and the physical meaning of each BIMF is unclear in the mode-mixing area ${ }^{[5-8]}$. Mode mixing problem is an inevitable but needs to be properly solve problem in EMD algorithm, which directly affect the EMD technique application in various fields. This paper discuss the reasons of mode mixing, and explain these reasons through some examples.

\section{EMD}

EMD is an adaptive and efficient method applied to decompose non-stationary and nonlinear signals into a series of IMFs. An IMF is a function that satisfies the two following conditions: (1) in the whole data set, the number of extreme and the number of zero-crossing must either equal or differ at most by one, and (2) at any point, the mean value of the envelope defined by the local maxima and the envelope defined by the local minima is zero. According to the assumption that any 
signal comprises IMFs of different scale, the EMD method can decompose a signal into a set of IMF components ${ }^{[4]}$. The EMD process of the signal $x(\mathrm{t})$ can be described as follows:

(1)Identify all the local maxima, and then connect all the local maxima by a cubic spline line as the upper envelope. Repeat the procedure for the local minima to produce the lower envelope. The upper and lower envelopes should cover all the data between them.

(2) The mean of upper and lower envelopes value is designated as $m_{1}(t)$, and the difference between the signal $x(\mathrm{t})$ and $\mathrm{m}_{1}(\mathrm{t})$ is $\mathrm{h}_{1}(\mathrm{t}): h_{1}(t)=x(t)-m_{1}(t)$;

(3) If $h_{1}(\mathrm{t})$ is an IMF, then $h_{1}(\mathrm{t})$ is the first component of $x(\mathrm{t})$.

(4) If $h_{1}(\mathrm{t})$ is not an IMF, $h_{1}(\mathrm{t})$ is treated as the original signal and repeat steps (1-3); we got:

$$
h_{11}(t)=h_{1}(t)-m_{11}(t)
$$

in which, $\mathrm{m}_{11}(\mathrm{t})$ is the mean of upper and lower envelopes value of $h_{1}(\mathrm{t})$.

(5) After repeated sifting process $K$ times, $h_{1 \mathrm{k}}(\mathrm{t})$ becomes an IMF, that is

Then, it is designated $c_{1}(\mathrm{t})=h_{1 \mathrm{k}}(\mathrm{t})$ as the first IMF component from the original data. $c_{1}(\mathrm{t})$ should contain the finest scale or the shortest period component of the signal.

(6) Separate $c_{1}(\mathrm{t})$ from $x(\mathrm{t})$, we could get:

$$
r_{1}(t)=x(t)-c_{1}(t)
$$

(7) $r_{1}(\mathrm{t})$ is treated as the original data, and repeat the above processes, the second IMF component $c_{2}(\mathrm{t})$ of $x(\mathrm{t})$ could be got.

(8) Let us repeat the process as described above for $n$ times, then n-IMFs of signal $x(t)$ could be got. Then,

$$
\begin{gathered}
r_{1}(t)-c_{2}(t)=r_{2}(t) \\
\vdots \\
r_{n-1}(t)-c_{n}(t)=r_{n}(t)
\end{gathered}
$$

The decomposition process can be stopped when $r_{\mathrm{n}}$ becomes a monotonic function, from which no more IMF can be extracted. By summing up (5) and (6), we finally obtain the residue $r_{\mathrm{n}}(\mathrm{t})$ is the mean trend of $x(\mathrm{t})$.

$$
x(t)=\sum_{j=1}^{n} c_{j}(t)+r_{n}(t)
$$

\section{The types and reasons of mode mixing problem}

(1)The jumping change of the decomposed signal result in mode mixing

The discontinuity of the decomposed signal is the major cause of the EMD mode mixing, such as noise interference, a high frequency wave discrete distribution in the original signal which lead the original signal to be a local high frequency signal, thus causes the point of local extreme value of the original signal distribution occur mutations, makes the envelope which was formed by the local extreme value point occur jump phenomena, resulting the IMF don't agree with the time scale, making the EMD mode mixing phenomenon occurred in the decomposition process, resulting the different frequency components in the original signals cannot be separated according to the characteristics of time scale effectively.

Here are illustrated with examples and analysis mode mixing caused by discontinuous signal example. Discontinuous signals is refers to in the time domain frequency discontinuity, such as:

Signals: $x_{1}(t)=a_{1} \sin \left(2 \pi f_{1} t\right), 0.05 \leq t \leq 0.1 ; x_{2}(t)=a_{2} \sin \left(2 \pi f_{2} t\right), 0.15 \leq t \leq 0.25$; 
$x_{3}(t)=\sin (20 \pi t) 0 \leq t \leq 0.3$

$x(t)=x_{1}(t)+x_{2}(t)+x_{3}(t)$, Amplitude: $a_{1}=0.2, a_{2}=0.4, a_{3}=1$; Frequency:

$f_{1}=150 \mathrm{~Hz}, f_{2}=50 \mathrm{~Hz}, f_{3}=10 \mathrm{~Hz}$ 。

The decomposed signal $x(t)$ is constitute by the high frequency with $150 \mathrm{HZ}$ and small amplitude with 0.2 , and the low frequency with $50 \mathrm{HZ}$ and small amplitude with 0.4 , and the low frequency with $10 \mathrm{HZ}$ and large amplitude with 0.4. The signal $x(t)$ was decomposed by EMD, the results were show by Fig.1.
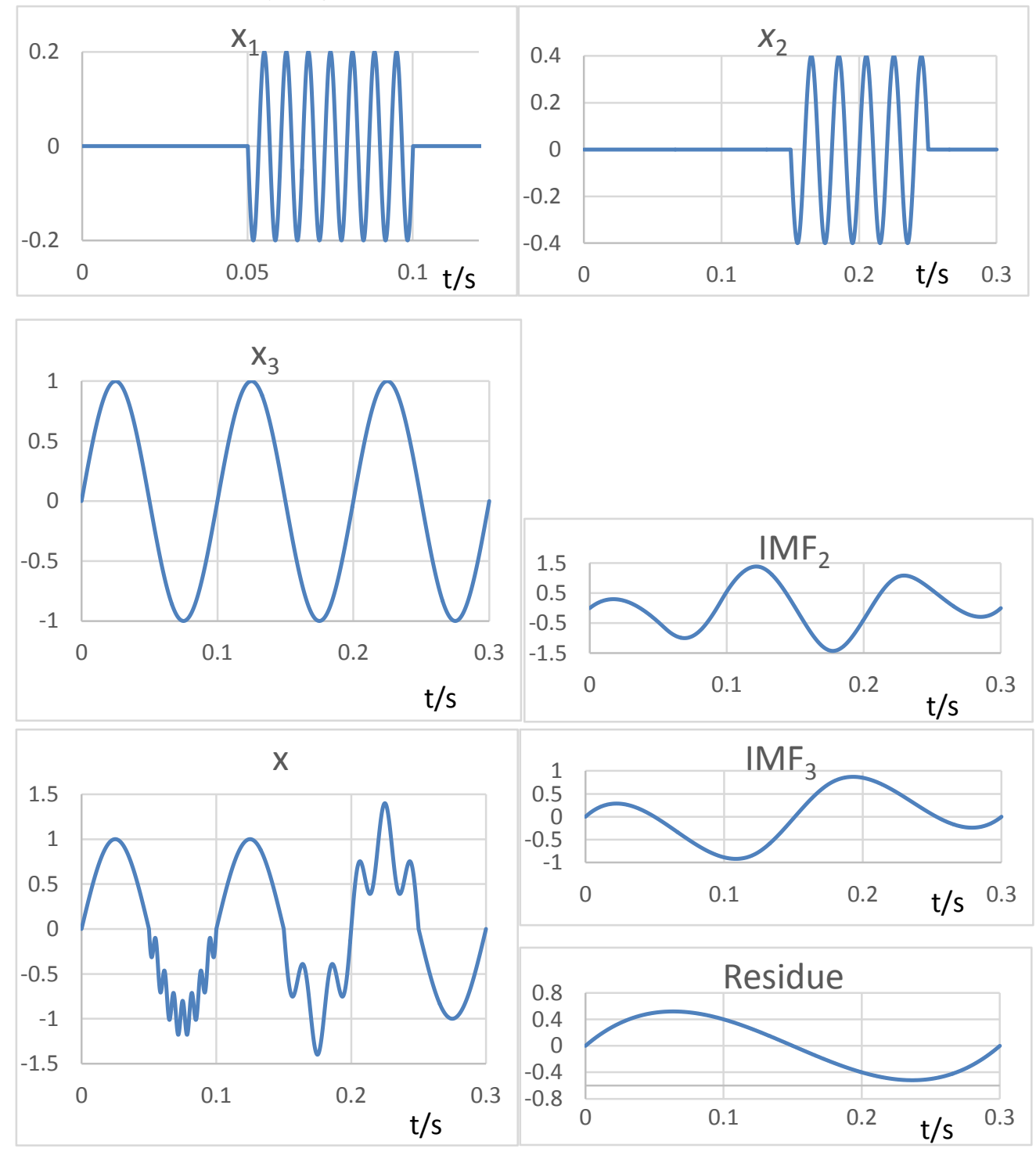

Fig.1. $x$ signal composition and the EMD decomposition results

There is a distinct mode mixing problem in the first IMF including signals with different frequencies (a high frequency $150 \mathrm{HZ}$, a medium frequency $50 \mathrm{HZ}$ and a low frequency $10 \mathrm{HZ}$ in this example) that show in IMF1. The IMF1 mode mixing phenomenon also affects the subsequent intrinsic mode function selection, the frequency characteristics of the scale is not clear in IMF2 which is obvious the mode mixing, and the mode mixing problem continues in the subsequent mode 
component. In physics, analyzes all signals' instantaneous frequency is meaningless, only when the signal is symmetric about the function of time, and the number of extreme value point and the number of passing zero are equal. So to be strictly say, the IMF2, IMF3 and IMF4 can only be described as the components of the EMD decomposition, and cannot be described as intrinsic mode functions, because it does not conform to the definition of intrinsic mode function, these components cannot be composed signal clear expression of the real physical process, so the mode mixing phenomenon violates the essence of EMD algorithm, this decomposition doesn't make any sense to significance, in order to analysis mode mixing phenomenon result by the decomposed signal intermittent clearly. The reason of why mode mixing can produce during the signal $x$ EMD decomposed process show in Fig.2.

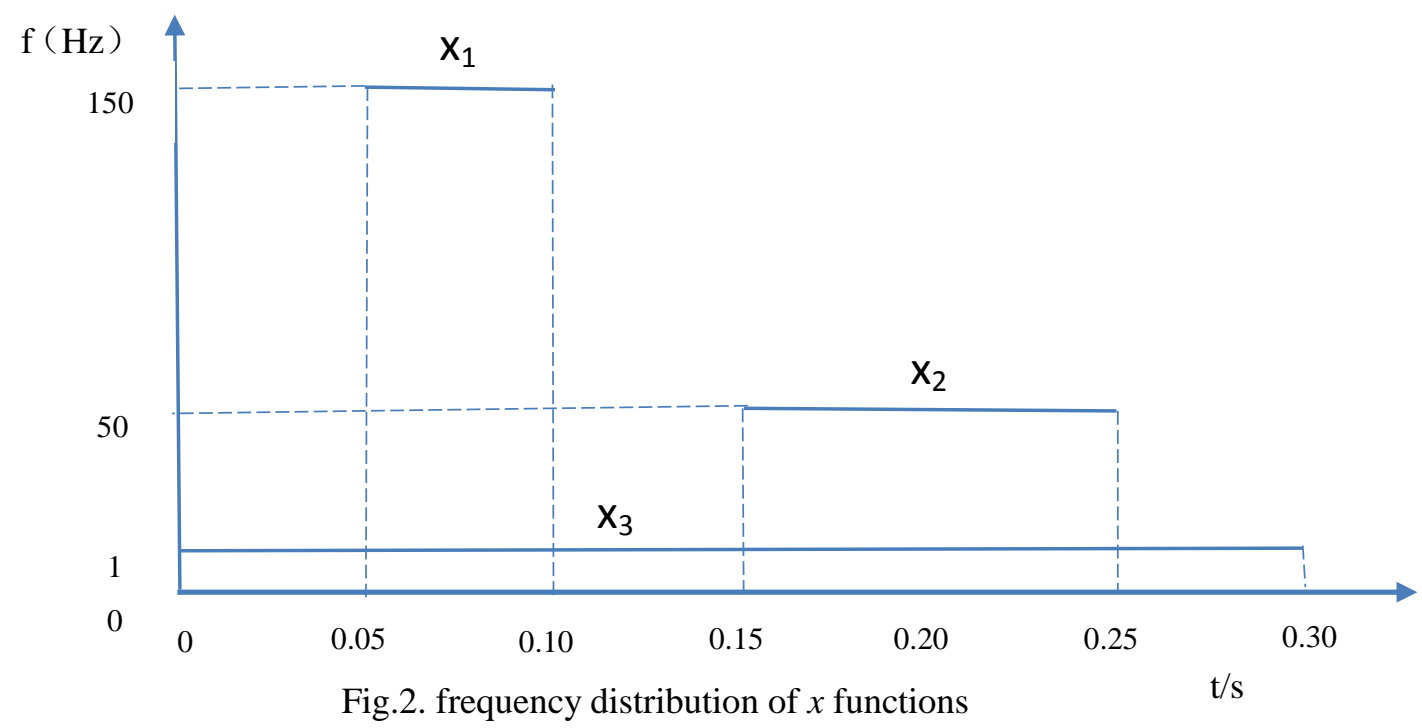

As shown in Fig.2, signal $x$ is more than one component, it is a synthesis signal by three kinds components of $x_{1}$ (frequency 10HZ), $x_{2}$ (frequency 50HZ) and $x_{3}$ (frequency 150HZ) respectively. Generally speaking, the overall result of the decomposition is to successively remove the highest frequency from a signal, under ideal condition, the signal $x$ decomposed by EMD to get three frequency high to low single components $x_{1} 、 x_{2}$ and $x_{3}$. According to the principle of EMD decomposition, in each iteration process, high frequency points is always emergence in the early decomposition of earlier intrinsic mode function. Therefore, for the signal $x$, on the whole time series, the first mode component should be:

$x_{3}(0 \leq t \leq 0.05) \rightarrow x_{1}(0.05<t \leq 0.10) \rightarrow x_{3}(0.10<t \leq 0.15)$, contrast to the EMD
$\rightarrow x_{2}(015<t \leq 0.25) \rightarrow x_{3}(0.25<t \leq 0.30)$

decomposition $\mathrm{IMF}_{1}$ results in Fig.1, the results consistent with the results according by the EMD decomposition principle. The EMD decomposition is an iterative loop, the mode mixing of first IMF will inevitably affect subsequent mode functions cannot correctly reflect its physical meaning.

(2) Mode mixing caused by amplitude and frequency relationship of the signal

Shen et al. ${ }^{[9]}$ point out that the original signal contains different signals with different frequency ratios and amplitude ratios can impact the EMD decompose results, here with the examples to illustrate:

Signal: $x(t)=a_{1} \sin \left(2 \pi f_{1} t\right)+a_{2} \sin \left(2 \pi f_{2} t\right)$ 
In the equation, $a_{1}$ and $a_{2}$ represent the amplitude of the two sinusoid which constitute of the signal $x(t), f_{1}$ and $f_{2}$ are the frequency of the two sinusoid, the literature (Shen et al., 2005) has given the EMD decomposition does not produce mode mixing conditions: $a_{1} f_{1}>a_{2} f_{2}$, 且
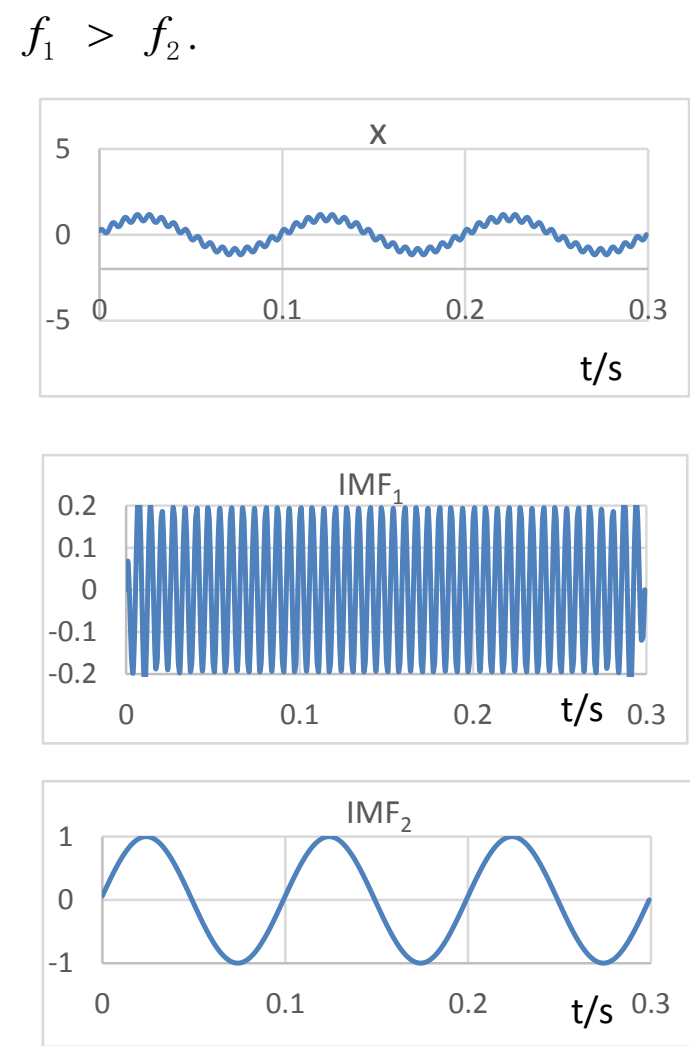

Fig.3. $x$ signal by EMD decomposition
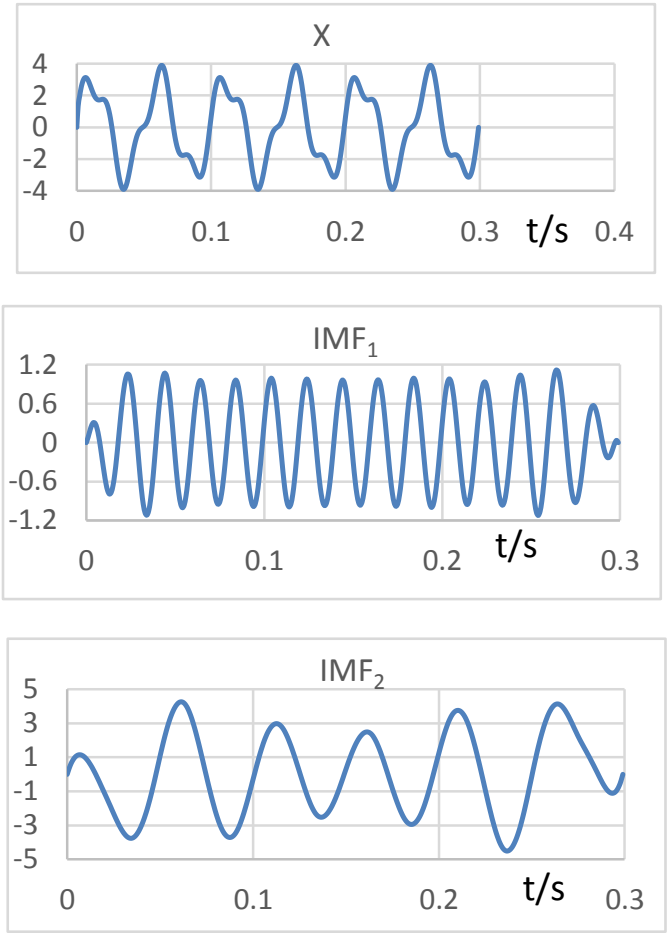

Fig.4. $x$ signal by EMD decomposition

Assuming that $a_{1}=0.2, f_{1}=150 \mathrm{~Hz}, a_{2}=1, f_{2}=10 \mathrm{~Hz}$, and $a_{1} f_{1}>a_{2} f_{2}$, the results in Fig. 3 show that the two component compositions in $x$ were separated smoothly.

Assuming that $a_{1}=12, f_{1}=50 \mathrm{~Hz}, a_{2}=3, f_{2}=20 \mathrm{~Hz}$, it don't satisfy the condition $a_{1} f_{1}>a_{2} f_{2}$, the EMD decomposition results produce the mode mixing IMF, the results show in Fig. 4. Contrast Fig. 3 and Fig. 4, we can find that the signal highest frequency parts to the signal lowest frequency parts selection by EMD on the premise condition of "local extremum points", if within a certain period of time, in synthetic signal some frequency components of the signal's contribution to the local extremum points number is zero, this is equivalent to the frequency signal within this period of time has been" swamped ", when the EMD decomposition wouldn't screening related to this information, this mode mixing problem is caused by the signal of the amplitude frequency relationship. With EMD decomposition by small intrinsic mode and greatly inherent mode superposition of signal, with small mode of the extremum points not apparent, the EMD can't effective screening out small intrinsic mode, screening is the basic component in the superposition of two or more than two intrinsic mode. 


\section{Conclusions}

To sum up, the reasons of mode mixing mainly include: high frequency weak signal in noise or contains discontinuous signal interference, result the extreme value point of the signal distribution confusion; mixed signal's amplitude and frequency relationship may lead to mode mixing. The essence of this phenomenon reason can be summed up in the EMD decomposition does not meet the global orthogonality.

\section{Acknowledgment}

The authors gratefully acknowledge the financial support from scientific research project of Hubei Provincial Education Department under Grant No. 20106401.

\section{References}

[1] Wieland, R., Dalchow, C., 2009. Detecting landscape forms using Fourier transformation and singular value decomposition (SVD). Computers \& Geosciences 35(7), 1409-1414.

[2] Pering, T., Tamburello, G., McGonigle, A., Hanna, E., Aiuppa, A., 2014. Correlation of oscillatory behaviour in matlab using wavelets. Computers \& Geosciences 70(9), 206-212.

[3] Khalil, M., Santos, F., Farzamian, M., El-Kenawy, A., 2015. 2-D Fourier transform analysis of the gravitational field of Northern Sinai Peninsula. Journal of Applied Geophysics 115, 1-10.

[4] Huang, N.E., Shen, Z., Long, S.R., Wu, M.C., Shih, H.H., Zheng, Q., Yen, N.C., Tung, C.C., Liu, H.H., 1998. The empirical mode decomposition and the Hilbert spectrum for nonlinear and non-stationary time series analysis. Proceedings of the Royal Society A 454 (1971), 903-995.

[5] Wu, Z., Huang, N., Chen, X., 2009. The multi-dimensional ensemble empirical mode decomposition method. Advances in Adaptive Data Analysis 1(3), 339-372.

[6] Sungkono, Bahri, A.S., Warnana, D.D., Monteiro Santos, F.A., Santosa, B.J., 2014a. Fast, simultaneous and robust VLF-EM data denoising and reconstruction via multivariate empirical mode decomposition. Computers \& Geosciences 67(2), 125-138.

[7] Chen, C., Jeng, Y., 2014. Two-dimensional nonlinear geophysical data filtering using the multidimensional EEMD method. Journal of Applied Geophysics 111, 256-270.

[8] Wu, Z., Huang, N., 2009. Ensemble empirical mode decomposition: a noise-assisted data analysis method. Advances in Adaptive Data Analysis 1(1), 1-41.

[9] Shen, G., Tao, L., Chen, Z., 2005. Theoretical research on empirical mode decomposition of multi-frequency signal and its application. Journal of Vibration Engineering 18(1): 91-94. 